\title{
A comparison of EISCAT and HF Doppler observations of a ULF wave
}

\author{
D. M. Wright, T. K. Yeoman, J. A. Davies \\ Department of Physics and Astronomy, University of Leicester, University Road, Leicester, LE1 7RH, UK. e-mail: dmw7@ion.le.uk \\ Received: 22 September 1997 / Revised: 9 January 1998 / Accepted: 16 January 1998
}

\begin{abstract}
Since the middle of 1995, an HF Doppler sounder has been running almost continuously in northern Norway, with the receiver at Ramfjordmoen and the transmitter at Seljelvnes. Concurrent operation of the EISCAT UHF radar in common programme (CP-1) mode has made it possible to study the ionospheric signature of a magnetospheric ULF wave. These are the first results of such wave signatures observed simultaneously in both instruments. It has been demonstrated that the observed Doppler signature was mainly due to the vertical bulk motion of the ionosphere caused by the electric field perturbation of the ULF wave and the first direct observational confirmation of a numerical simulation has been achieved. The wave, which was Alfvénic in nature, was detected by the instruments $8^{\circ}$ equatorward of the broad resonance region. The implications for the deduced wave modes in the ionosphere and the mechanism producing the HF Doppler variations are discussed.
\end{abstract}

Key words. Magnetosphere-ionosphere interactions · MHD waves and instabilities · Radio science ·

Ionospheric physics

\section{Introduction}

Ultra-low-frequency (ULF) waves are an important coupling mechanism between the magnetosphere and the ionosphere since they transfer both energy and momentum. These interactions are most significant in

Correspondence to: D. M. Wright

Presented at the Eighth International EISCAT Workshop, Leicester, UK, June 1997 the high-latitude ionosphere, where the magnetosphereionosphere interaction is strongest. The waves also act as an important diagnostic of magnetospheric morphology and dynamics. The ionosphere determines the boundary conditions (e.g. Yeoman et al., 1990) for magnetospheric magnetohydrodynamic (MHD) wave modes and hence controls the transfer of energy and momentum. It also modifies the magnetospheric wave signature, leading to rotation and attenuation of the wave magnetic signature detected on the ground (e.g. Hughes and Southwood, 1976; Hughes, 1983). The ionospheric signature of ULF waves is thus an important topic of study.

Up to the present the principal instruments for the investigation of the ionospheric signatures of ULF waves have been coherent-scatter radars. There are many reports of the ionospheric signatures of field line resonances in both the E-region, using VHF radar data (e.g. Walker et al., 1979; Yeoman et al., 1990) and in the F-region, using high-frequency (HF) radar data (e.g. Ruohoniemi et al., 1991). These waves generally exhibit small effective azimuthal wave numbers $(m)$ typically in the range $0-20$, which is equivalent to azimuthal wavelengths of greater than $685 \mathrm{~km}$ at the latitude of Troms $\varnothing$ $\left(69.6^{\circ} \mathrm{N}\right)$, although observations up to $m=40$ have occasionally occurred (e.g. Fenrich et al., 1995). Nonresonant ULF wave features have also been observed by VHF radars (e.g. Allan et al., 1982, 1983). Fenwick and Villard (1960) first suggested that geomagnetic variations might be associated with observed shifts in the frequency of ionospherically reflected radio signals recorded with an HF Doppler sounder. Since then, short-period oscillations in the frequency of HF signals have been correlated with magnetospheric ULF pulsations measured by ground magnetometers (e.g. Jacobs and Watanabe, 1966; Klostermeyer and Röttger, 1976; Tedd et al., 1989; Menk, 1992). Recently, Wright et al. (1997) presented a detailed study of ULF wave signatures observed by a new sounder at high latitudes (see Sect. 2.1). Models of the mechanisms involved in generating the ionospheric signatures observed by HF Doppler 
sounders have been described by Poole et al. (1988), Sutcliffe and Poole $(1989,1990)$ and Sutcliffe (1994).

HF Doppler sounders offer an important experimental technique for investigating the ionospheric signatures of magnetospheric ULF waves. This type of radio diagnostic offers the ability to select the height at which the ionosphere is probed by changing the sounding frequency, as well as providing measurements of the ionosphere with high spatial and temporal resolution. A new sounder, known as the Doppler Pulsation Experiment (DOPE), has recently been constructed in Leicester, UK, and was deployed in May 1995 near Tromsø, Norway (geographic: $69.6^{\circ} \mathrm{N} 19.2^{\circ} \mathrm{E}$; geomagnetic: $\left.67.0^{\circ} \mathrm{N} 117.0^{\circ} \mathrm{E} ; L=6.3\right)$. It is located close to the European Incoherent Scatter (EISCAT) radar facility (e.g. Rishbeth and Williams, 1985) which enables comparison with EISCAT data from suitable common and special programmes, with the aim of investigating the ionospheric boundary conditions for MHD wave modes in the magnetosphere, studying the height profile of ULF wave signatures, and comparing observations with existing theoretical models and measurements made with other ground-based instruments. A limited number of observations of the ionospheric signatures of ULF waves with the EISCAT radar have been reported by Crowley et al. $(1985,1987)$ and Glangeaud et al. (1985).

This paper presents the first observations made by DOPE in conjunction with the EISCAT UHF radar. The simultaneous detection of a Pc5 pulsation at the ground and its signature in the ionosphere at high latitudes has allowed the vertical and horizontal structure of the ULF wave to be investigated quantitatively. The observations are interpreted as a field line resonance and the observed Doppler signature is demonstrated to be due to the vertical component of an $\boldsymbol{E} \times \boldsymbol{B}$ motion in the ionosphere, caused by the incident ULF wave.

\section{Instrumentation}

\subsection{The high-latitude Doppler sounder}

The DOPE sounder consists of a frequency stable transmitter (Chapman, 1995, 1997b) and a receiver (Chapman, 1997a) which have a ground-separation of about $50 \mathrm{~km}$, giving a near vertical radio path roughly along a magnetic meridian (Wright et al., 1997). A fixedfrequency $(4.45 \mathrm{MHz})$ continuous wave signal is radiated and, after reflection in the F-region ionosphere, received at the ground. The Doppler technique works on the principle that variations in the refractive index or bulk motion of the plasma along the path of the radio wave cause small shifts in the received frequency, due to changes in the phase path of the wave. Thus, the frequency shift, $\Delta f$, is given by

$\Delta f=-\frac{1}{\lambda} \frac{\mathrm{d} P}{\mathrm{~d} t}$

where $P$ is the phase path of the signal, $\lambda$ is the wavelength and $t$ is time. The measured Doppler shifts, which are typically less than $1 \mathrm{~Hz}$, can thus indicate the characteristics of waves affecting the ionospheric plasma in the region being sounded. In the case where the refractive index does not vary with time, this can be interpreted as an equivalent vertical motion of the reflection point with a velocity, $v$, using, for a vertical incidence sounder, the relation

$$
\Delta f=-2 \frac{v}{c} f \text {, }
$$

where $c$ is the speed of light and $f$ is the sounding frequency.

The spatial resolution of a sounder such as this is determined by the area over which it integrates data. This is given, to a first approximation, by the region of specular reflection of the HF radio wave from the ionosphere which is equal to the area of the first Fresnel zone of the reflecting plane of the ionosphere (see Appendix). The scale size for the sounder has thus been determined to be $\sim 4 \mathrm{~km}$. When compared to the scale size of the integration area of ground magnetometers $(\geq 120 \mathrm{~km}$; Hughes and Southwood, 1976) and VHF coherent-scatter radars $(15-45 \mathrm{~km})$, it is clear that a Doppler sounder offers ionospheric observations with high spatial resolution. The EISCAT incoherent-scatter radar has a beam width of the order of $2 \mathrm{~km}$ at F-region heights. When considering a real, and therefore non-planar, ionosphere, the broad antenna pattern of the Doppler sounder means that it is possible that the received Doppler signal may contain contributions from more than one ionospheric interaction region.

\subsection{Data acquisition and analysis}

The Doppler sounder receiver system consists of a twinchannel receiver and a PC employed to control receiver operations and to log data on a digital audio tape (Wright, 1996). The signal is received on a pair of crossed active dipole antennas and an $\mathrm{O}-\mathrm{X}$ mode discriminator enables $\mathrm{O}$ - and $\mathrm{X}$-mode signals to be resolved on channels 1 and 2 of the receiver, respectively (Chapman, 1997a). The receiver mixes the signal input frequency down to a baseband level which represents the offset from the diagnostic frequency of $4.45 \mathrm{MHz}$. The receiver output is filtered with a cut-off of $15 \mathrm{~Hz}$ at the $3-\mathrm{dB}$ level. So far as the authors are aware, this is the first time continuous $\mathrm{O}$ - and $\mathrm{X}$-mode observations of ULF wave signatures have been made at high latitudes. A digital ionosonde located at the geomagnetically midlatitude station of Halley, Antarctica, has employed Oand $\mathrm{X}$-mode discrimination to make observations of the ionospheric signatures of Pc3-4 pulsations (Jarvis and Gough, 1988) and of Pc1 pulsations (Jarvis and Morrison, 1997). However, in each case the number of events presented was small. In addition, low-latitude observations utilising $\mathrm{O}-\mathrm{X}$ discrimination have been reported by Sutcliffe and Poole (1984) in their study of Pc3 signatures and, more recently, by Menk et al. (1995). 
The data, which are sampled at the receiver output at a rate of $40 \mathrm{~Hz}$, undergo spectral analysis employing a fast Fourier transform (FFT) routine in order to produce a "Doppler trace" such as those reproduced in this paper. The temporal resolution of these data is $12.8 \mathrm{~s}$ which is a result of consecutive blocks of 512 samples being Fourier analysed. Spectral components with values above some threshold (usually $50 \%$ of the peak value) are included on the Doppler trace and these represent the diagnostic signal frequency shift.

\subsection{UHF incoherent-scatter measurements}

The EISCAT UHF (ultra high frequency) radar (Rishbeth and Williams, 1985) is an incoherent-scatter radar which operates at a frequency around $931 \mathrm{MHz}$. The system consists of three $32-\mathrm{m}$ dishes: one transmitreceive site at Tromsø and receive-only dishes at Sodankylä, Finland and Kiruna, Sweden. Tristatic measurements of ion flow velocity enables the $\boldsymbol{E} \times \boldsymbol{B}$ velocity vector in the F-region to be determined. An EISCAT special programme, SP-UK-DOPE, has been written for this experiment which is similar to the CP-1 common programme. Both utilise an alternating code pulse scheme at low altitude in the E- and lower Fregions and a long-pulse scheme for F-region and topside observations. The former type of pulse coding provides the higher spatial resolution required for measurements in the E-region (e.g. Davies et al., 1997).

The combined EISCAT UHF and DOPE sounder experimental set-up is illustrated in Fig. 1. A constant pointing direction of EISCAT allows measurements to be made with a high time resolution. SP-UK-DOPE has the flexibility to allow the experimenter to quickly

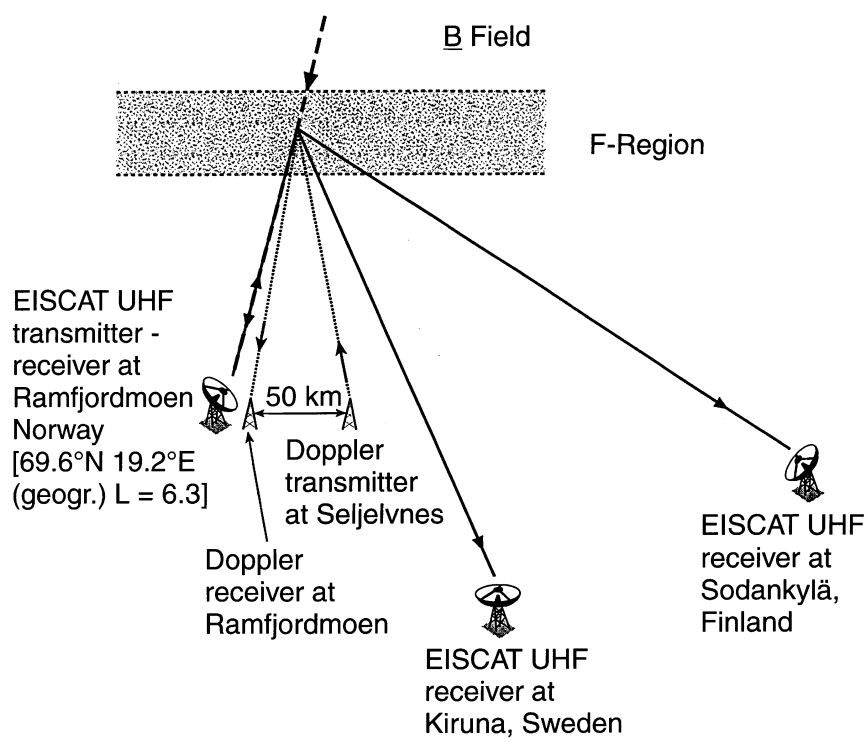

Fig. 1. A schematic representation of the instrumental arrangement for the EISCAT SP-UK-DOPE experiment. The DOPE sounder and the EISCAT UHF radar make simultaneous measurements. Also located at Tromsø are a Dynasonde and one of the IMAGE ground magnetometer stations change the intersection altitude of the radar, i.e. the height at which the tristatic flow velocity is measured. The intersection height can therefore be set to a position where the signal-to-noise ratio of the returned signals is high, thus minimising the amount of post-integration necessary on the data. It is desirable to match the reflection height of the HF sounder wave with the interaction height of EISCAT (see Fig. 1) so that any ULF wave effects are monitored at the same part of their vertical profile with both instruments. The height of the HF Doppler wave reflection point can be determined, in near real time, by either inverting a recent ionogram measured by the Tromsø Dynasonde, a digital ionosonde, or by examining the electron density information of the local ionosphere provided by EISCAT power profile measurements. It should be noted that EISCAT CP-1 data have been employed in this study.

\subsection{Ground magnetometers}

In addition to the HF Doppler and EISCAT data presented in this paper, data from the IMAGE (Lühr, 1994) ground magnetometer stations in northern Scandinavia are included. These data are given in a geographic (XYZ) coordinate system and have a time resolution of $10 \mathrm{~s}$. The resolution of the magnetometer at Tromsø (TRO) is $1 \mathrm{nT}$ and therefore for a ground magnetic signature to be measured in association with ionospheric Doppler observations a ground amplitude of at least $4 \mathrm{nT}$ peak-to-peak is required. Spatially separated stations offer the possibility to determine the latitudinal phase change of a ULF wave as well as its azimuthal phase change, characterised by the effective azimuthal wave number, $m$, the number of degrees of change in wave phase per degree of longitude on the Earth's surface. Due to the way in which ground magnetometers integrate information over an area with a scale length of the order of the E-region height (Hughes and Southwood, 1976), phase mixing of signals from adjacent regions in the ionosphere results in the attenuation of waves with high $m$-values (corresponding to small azimuthal scale lengths) observed on the ground.

\section{Results}

This section presents data associated with a ULF wave which occurred on 13 February 1996 and was detected simultaneously in the ionosphere by the DOPE sounder and the EISCAT UHF radar and at the ground by IMAGE magnetometer stations. The top two panels of Fig. 2 illustrate the event as it was observed by the DOPE sounder in both the O- and X-mode signals. A series of wave cycles are clear in the data which start at some time before $10.40 \mathrm{UT}$ and continue until about 13.00 UT. The Doppler data outside of these times are noisy because the received signal amplitude was low due to increased absorption or because the signals had 


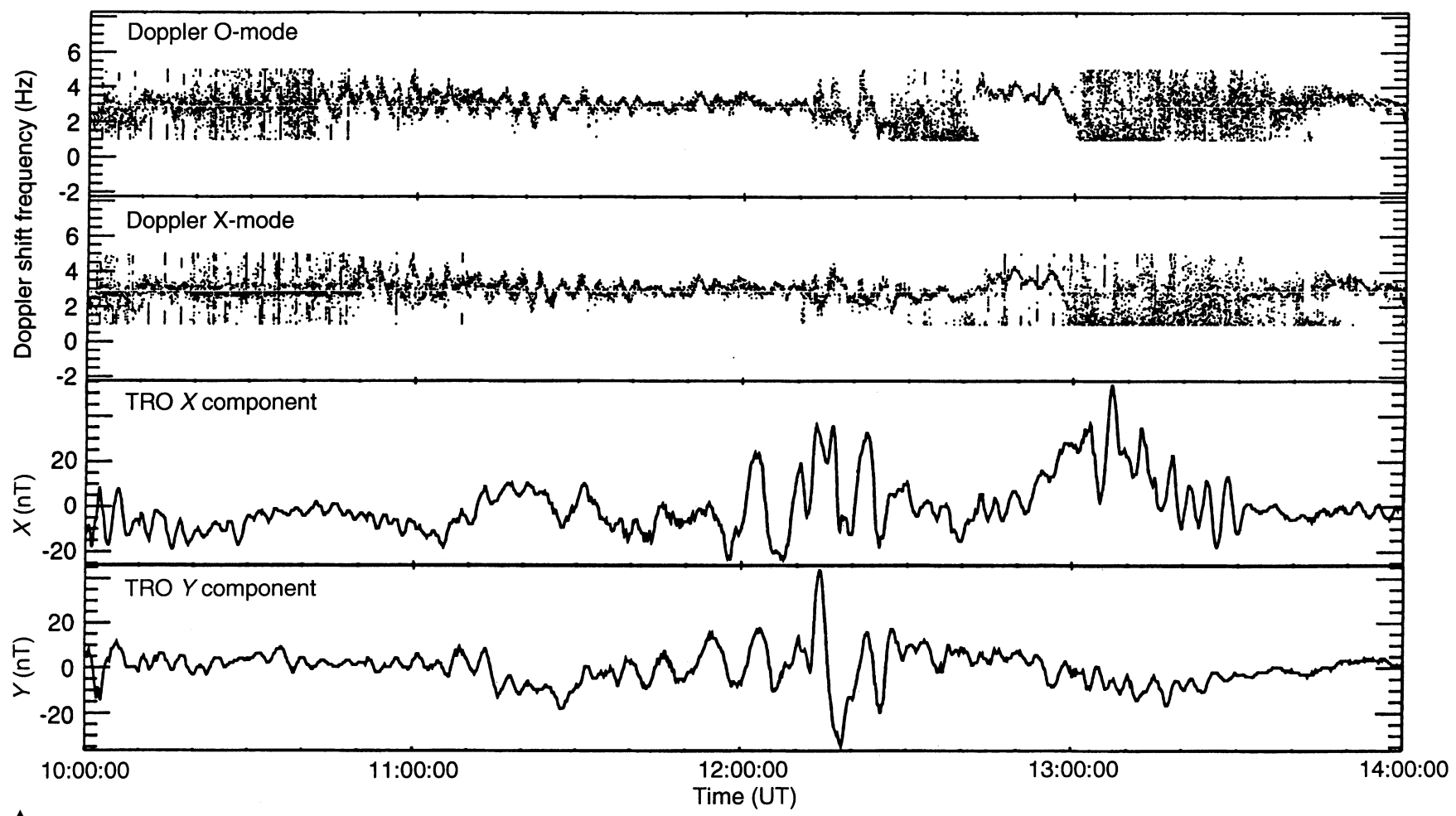

Fig. 2. HF Doppler and magnetometer data for the ULF wave event on 13 February 1996. The panels display (from the top down): Doppler O-mode frequency shift, X-mode frequency shift, TRO $X$ component and TRO $Y$-component. The sampling interval of the Doppler data is 12.8 and $10.0 \mathrm{~s}$ for that from the magnetometers. The Doppler data are plotted above a threshold of $50 \%$ of the amplitude of the main spectral component for each FFT
Fig. 3a-c. Time-series of the a peak traces for the $\mathrm{O}$ - and $\mathrm{X}$-mode signatures, b TRO magnetometer $X$ - and $Y$-components and $\mathbf{c}$ the northward and eastward components of the field perpendicular velocity observed by the EISCAT UHF radar for the interval 11.00-11.40 UT on 13 February 1996. Data are filtered to exclude periods outside the range $60-300 \mathrm{~s}(\mathrm{a}, \mathrm{b})$ and $120-400 \mathrm{~s}(\mathrm{c})$. Note that the ordinate axis is a relative scale with arbitrary offsets applied to each trace

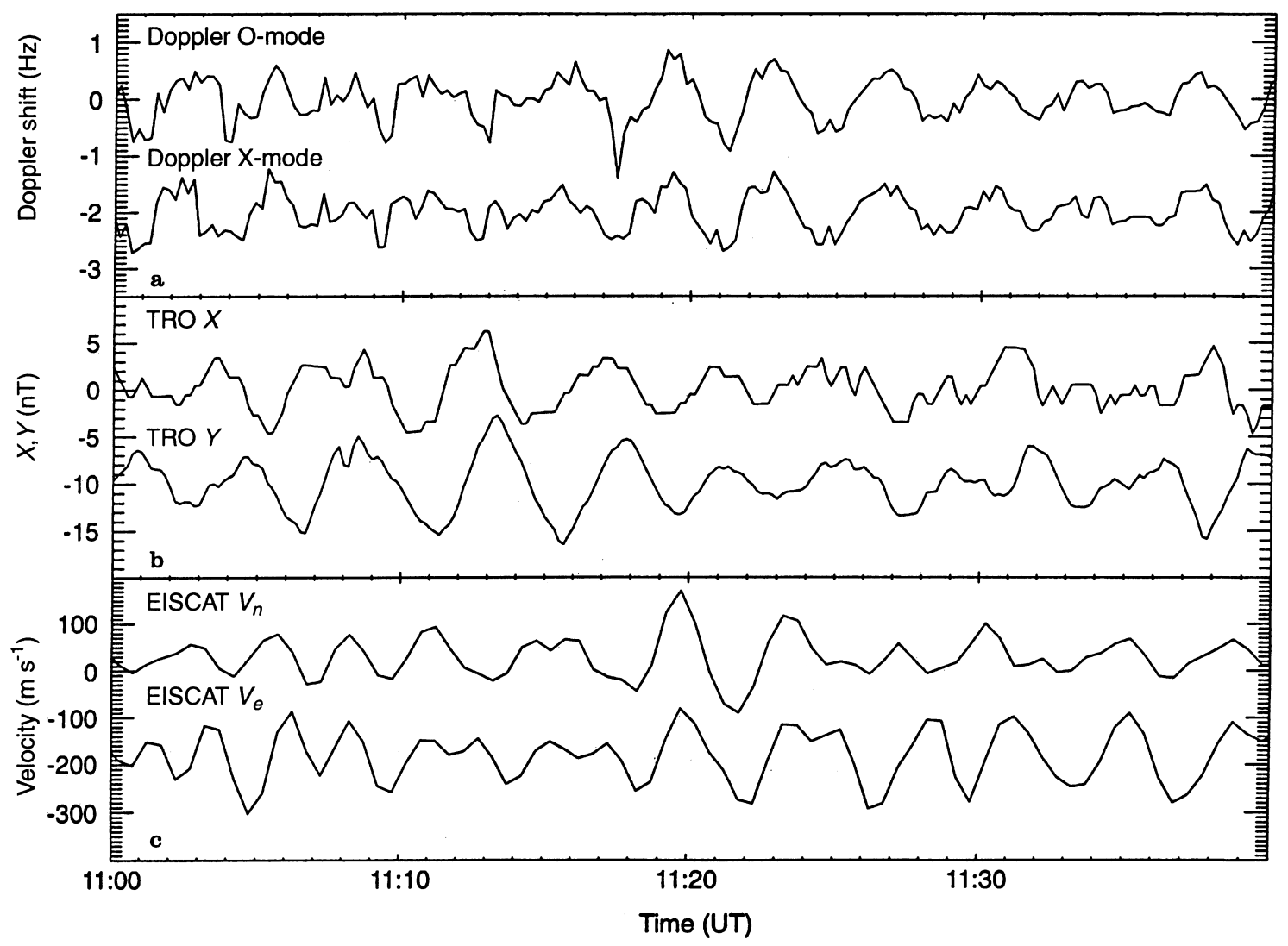


penetrated the ionosphere (the transmitted frequency was close to the local critical frequency of about 4.5 $\mathrm{MHz}$ and the reflection height of the O-mode wave was about $210 \mathrm{~km}$ ) and thus the wave cannot be seen at these times.

The lower two panels of Fig. 2 display the $X$ - and $Y$ components measured by the TRO magnetometer. There is a considerable amount of wave activity present in the ground magnetometer data during this interval. Figure 3 depicts a small section of this interval, selected because of the clarity of the Doppler signature. The Doppler time-series (Fig. 3a) are formed by selecting the peak component from each sequential spectrum (see Wright et al., 1997). This is necessary in order to make a frequency analysis of the wave possible. $X$ - and $Y$ component TRO magnetometer data for the same interval are reproduced in Fig. 3b. Concurrent with these observations, the EISCAT UHF radar was running the common programme $\mathrm{CP}-1$ and making tristatic measurements of the plasma flow velocity at an altitude of $278 \mathrm{~km}$. The EISCAT observations are thus made at a higher altitude than those from the DOPE sounder. Figure $3 \mathrm{c}$ illustrates the field perpendicular components of this velocity resolved into the northward $\left(v_{n}\right)$ and eastward $\left(v_{e}\right)$ directions.

In order to remove the high-frequency noise and long-period trends, all of the data in Fig. 3a, b have been filtered to exclude signatures outside the period range 30-400 s. However, the EISCAT measurements (Fig. 3c) have been filtered in the narrower range of 120-400 s, since these data contain more high-frequency noise. It is clear that there is a wave in all six time-series which is of similar period. Important points to note here are that the $\mathrm{O}$ - and $\mathrm{X}$-mode signatures are in phase and that there is a large phase difference (almost $180^{\circ}$ ) between the ground magnetic and the ionospheric signatures. In particular, the relative phase between the TRO $Y$-component and the Doppler O-mode signature, $\Delta \varphi_{\mathrm{YO}}$, is $\sim 160^{\circ}$ with $b_{y}$ leading. This value will be useful for a comparison with modelling work later in this study. Displayed in Fig. 4 are the normalised Fourier power spectra for each of the six time-series in Fig. 3. They indicate that, within the resolution of the spectra $(0.67 \mathrm{mHz})$, a wave exists in each with a period of $250 \mathrm{~s}$ (a frequency of $4 \mathrm{mHz}$ ) which is in the frequency range of a Pc5 pulsation (e.g. Jacobs et al., 1964). Small spectral peaks are also apparent at 6,8 and $10 \mathrm{mHz}$ which might relate to harmonics of the fundamental wave.

In order to determine the characteristics of the observed ULF wave, data from 17 magnetometers in the IMAGE network, which are located throughout Scandinavia, were examined. The stations range in geomagnetic latitude (see Lühr, 1994; also information on the internet at http://www.geo.fmi.fi/image/) from $56^{\circ} \mathrm{N}$ (Nurmijarvi, NUR) to $76^{\circ} \mathrm{N}$ (Ny Ålesund, NAL). Figure 5 displays $X$-component data, which again have been filtered in the range 30-400 s, for these stations. The same wave signature is evident in all time-series and exhibits a peak in amplitude at the higher-latitude

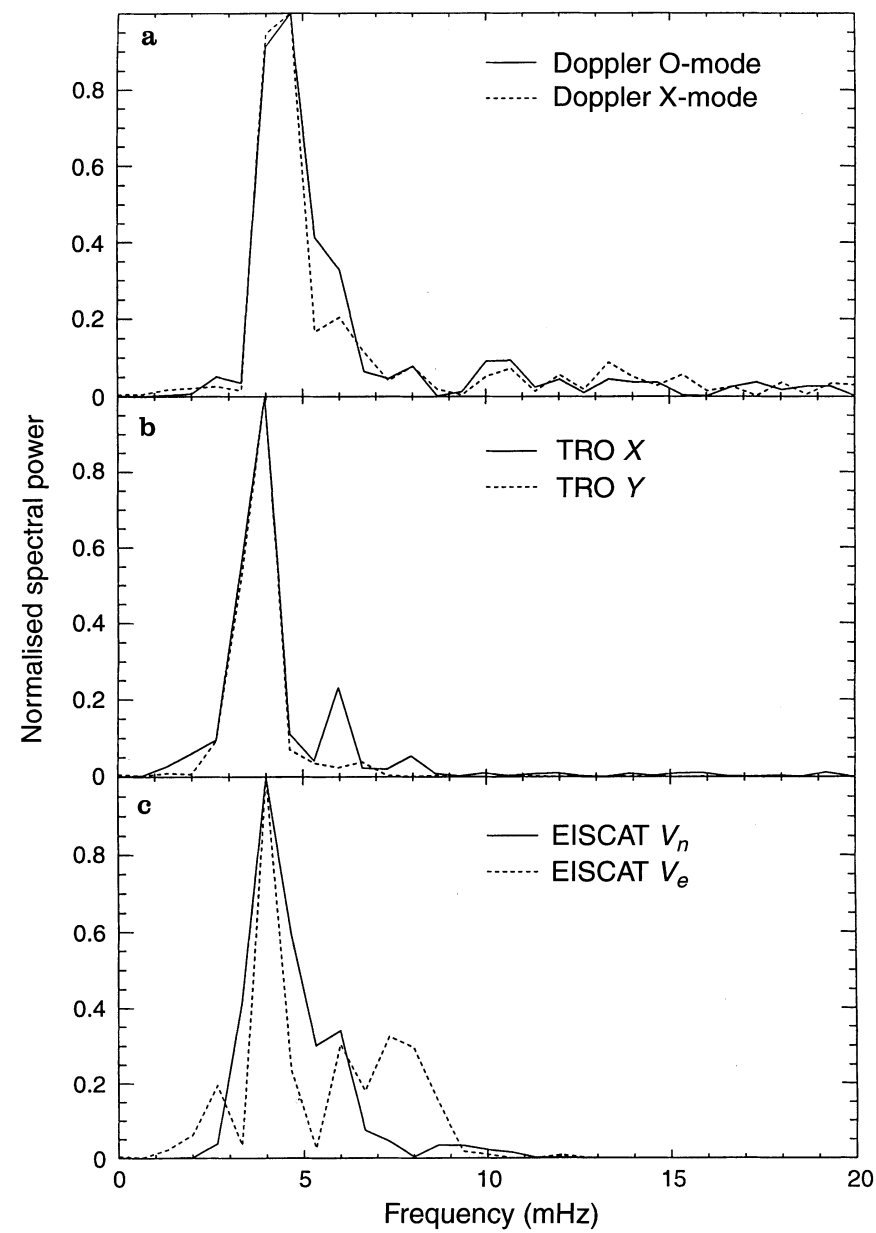

Fig. 4a-c. Normalised Fourier power spectra for each of the timeseries in Fig. $3 \mathrm{a}-\mathrm{c}$ for the interval 11.03-11.28 UT

stations. Parts a and b of Fig. 6 show respectively the latitudinal profiles of the spectral power of the $4 \mathrm{mHz}$ wave and the associated Fourier phase of this component obtained for each station. For reference, the location of Tromsø (TRO) is marked by a dashed line. The amplitude profile exhibits a clear, broad peak maximising around $75^{\circ} \mathrm{N}$ (geomagnetic) whilst the phase of the ULF wave changes through $180^{\circ}$ across the region of peak amplitude, remaining almost constant across the lower latitude stations below $71^{\circ} \mathrm{N}$. Figure 6 is thus demonstrating the characteristic signature of a field line resonance (e.g. Orr, 1984). However, the resonance region, measured at full-width half-maximum extends over about $5^{\circ}$ of latitude $(\sim 500 \mathrm{~km}$ in ground range) and might be considered large when compared to some previous observations [e.g. Yeoman et al. (1997) describe a field line resonance in HF radar data with a scale size of only $60 \mathrm{~km}]$. The $m$-number of the wave in this study was determined using the IMAGE station's $Y$-component data for the same interval as in Fig. 5, and is 2.9 (travelling westwards). This is consistent with the $m$-numbers of field line resonances determined from previous high-latitude radar observations (e.g. Yeoman et al., 1990; Fenrich et al., 1995). 


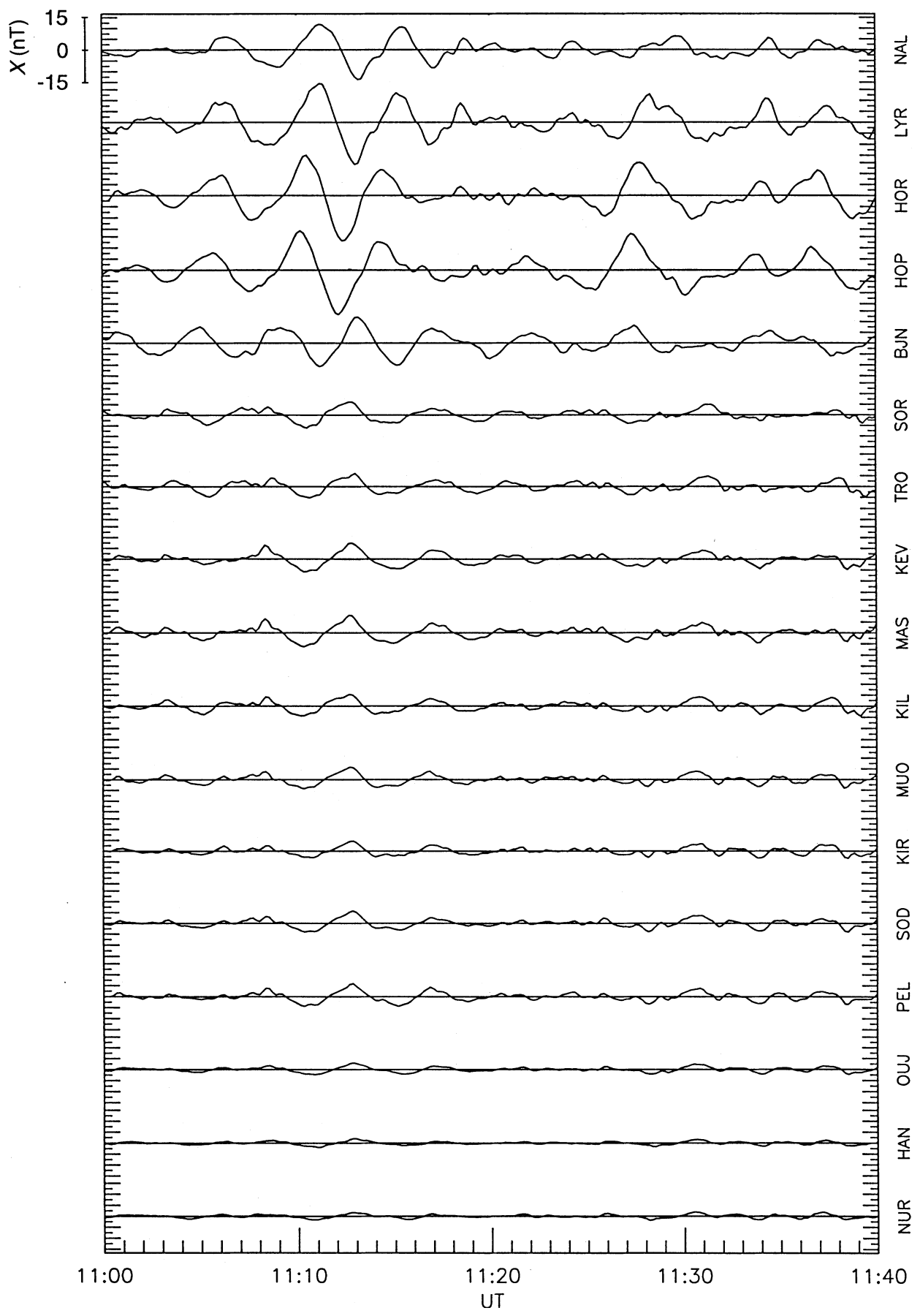

Fig. 5. X-component magnetometer data from 17 ground stations in the IMAGE network. Station geomagnetic latitudes range from $56^{\circ} \mathrm{N}$ (NUR) to $76^{\circ} \mathrm{N}$ (NAL). All data have been filtered to exclude periods outside the range $30-400 \mathrm{~s}$

\section{Discussion}

\subsection{The observations}

Figure $6 \mathrm{a}, \mathrm{b}$ clearly demonstrates that the ground magnetic signature of the event described in Sect. 3 is one of a classic field line resonance (Southwood, 1974; Orr, 1984). However, the resonant region is large with a scale size of around $500 \mathrm{~km}$. The fact that the $\mathrm{O}$ - and Xmode Doppler signatures, which are measurements of two latitudinally separated positions (Wright et al., 1997), are in phase confirms the magnetometer observations that Tromsø is located well away from the resonance region where the phase of the ULF wave changes rapidly with latitude. The period of the wave at $250 \mathrm{~s}$ is in agreement with the range of periods identified by Poulter et al. (1984) at high latitudes in STARE radar data. The $m$-number of the wave being 2.9 is consistent with previous radar observations of field line resonances at similar latitudes (Yeoman et al., 1990; Ruohoniemi et al., 1991).

The simultaneous observation of the ULF wave by the EISCAT UHF radar further confirms that HF Doppler sounders are sensitive detectors of the ionospheric signatures of ULF waves (e.g. Klostermeyer and Röttger, 1976; Menk, 1992; Wright, 1996; Wright et al., 1997). It also demonstrates the usefulness of the 


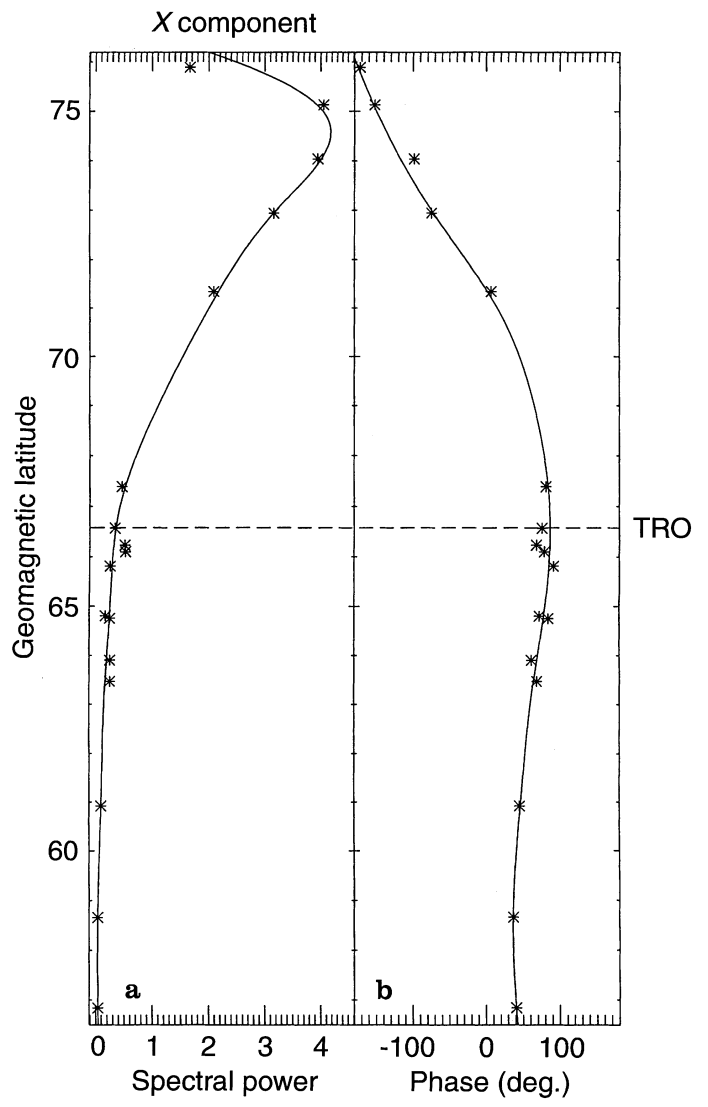

Fig. 6a, b. Latitudinal a Fourier spectral power and $\mathbf{b}$ phase profiles of the $4 \mathrm{mHz}$ spectral component derived from the $X$-component magnetometer data illustrated in Fig. 5. The dashed line indicates the location of the Tromsø (TRO) magnetometer

EISCAT CP-1 and SP-UK-DOPE programmes for supporting and validating the observations of ULF waves by HF Doppler sounders (e.g. Wright, 1996; Wright et al., 1997).

\subsection{Comparison of the data with modelling work}

Poole et al. (1988) presented models defining the mechanisms which may relate an incident ULF wave to its associated ionospheric Doppler signature at midlatitudes. They considerably extended previous models which considered only a motor effect (Rishbeth and Garriot, 1964; Klostermeyer and Röttger, 1976), where the electric field associated with a geomagnetic pulsation drives an $\boldsymbol{E} \times \boldsymbol{B}$ bulk drift of plasma which, due to the inclination of the field, has a vertical component. This is applicable to Alfvén mode waves with their associated ionospheric magnetic and electric field perturbations. Poole et al. (1988) accounted for changes in refractive index along the radio wave path and also included terms to compensate for magnetic field compression in the ionosphere.

Examination of electron density and electron temperature measurements by EISCAT indicates that the background conditions were steady at the time of the event presented here and not significantly modulated at the wave frequency. In addition, the $K_{p}$ index had a magnitude of 2 . It seems reasonable, therefore, to assume that local source and loss processes were negligible during this interval. By neglecting these processes for the ionospheric plasma, the model of Poole et al. (1988) was separated into three main mechanisms. The "magnetic" mechanism accounts for changes in the refractive index due to changes in the magnetic field intensity, requiring no bodily movement of electrons. The "advection" mechanism describes the change in the refractive index caused by the vertical motion of electrons under the influence of an eastward directed pulsation electric field. The presence of compressional mode waves in the ionosphere, and also the conversion of the incident Alfvén wave into an evanescent fast mode wave (which is subsequently detected at the ground) in the ionospheric E-region results in compression of the magnetic field, leading to changes in the local plasma density and, hence, the refractive index. This is the "compression" term, in the nomenclature of Poole et al. (1988) and Sutcliffe and Poole (1989, 1990).

The three components each contribute vectorially to the total calculated Doppler velocity. The phase of the mechanisms relative to the magnetic signature on the ground is a critical part of the model (Poole et al., 1988; Sutcliffe and Poole, 1989, 1990; Sutcliffe, 1994). The largest contributions to the Doppler velocity are due to the advection and compression terms. However, it is important to remember that currently the models discussed here apply to mid-latitudes and this paper is making a tentative extrapolation to high-latitude conditions (see also Wright, 1996; Wright et al., 1997). At Fregion heights the phase of these terms respectively lag and lead the $b_{y}$ component on the ground. Wright et al. (1997) have suggested that signatures exhibiting relative phases $\Delta \varphi_{\text {YO }}$ in the range $90^{\circ}-120^{\circ}$ ( $b_{y}$ leading) were a result of the advection mechanism as proposed by Poole et al. (1988) and, later, Sutcliffe and Poole (1989, 1990) and Sutcliffe (1994). The event presented here has a $\Delta \varphi_{\text {YO }}$ of $\sim 160^{\circ}$ with $b_{y}$ leading which implies that it would also be primarily the result of the advection mechanism. Figure 7 displays the $\mathrm{O}$ - and $\mathrm{X}$-mode Doppler velocities associated with the event, calculated using Eq. (2). Also included (Fig. 7c) is the vertical component of the northward field perpendicular velocity $\left(v_{n}(z)\right)$ measured by EISCAT, which is simply $(\boldsymbol{E} \times \boldsymbol{B})_{z}-$ the advection mechanism defined in the modelling work. The velocity signatures agree closely in amplitude, phase and wave period. The fact that the velocities match demonstrates that the advection mechanism is likely to be almost solely responsible for creating the ionospheric signature of the ULF wave presented here. Compressional wave modes are generally expected to have small electric fields associated with them in the ionosphere (Kivelson and Southwood, 1988; Yeoman et al., 1990), however the EISCAT and HF Doppler observations presented here exhibit a significant electric field in the ionosphere, which supports the belief that this event is Alfvénic. If the field lines were responding to an 


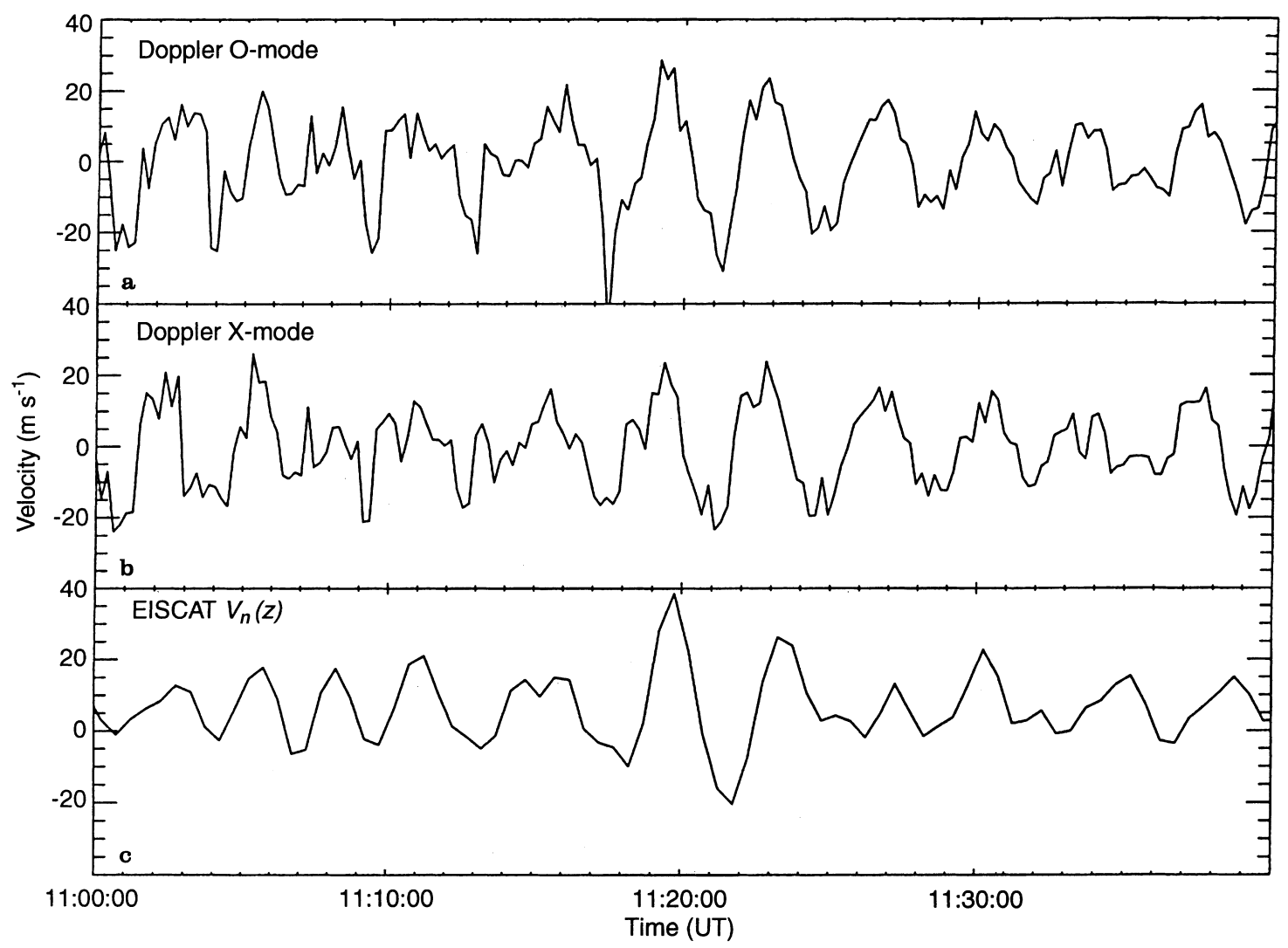

Fig. 7a-c. Velocities derived, using Eq. (2), from the Doppler a Omode and $\mathbf{b} \mathrm{X}$-mode signals and $\mathbf{c}$ the vertical component of the northward field perpendicular velocity measured by EISCAT for the interval 11.00-11.40 UT on 13 February 1996. Data have been filtered to exclude periods outside the range $30-400 \mathrm{~s}(\mathrm{a}, \mathrm{b})$ and $120-400 \mathrm{~s}(\mathrm{c})$. In each case the ordinate axes display relative scales incoming fast mode wave, it would be expected that a significant contribution to the ionospheric signature would be the result of a downgoing fast mode wave (since the Alfvénic contribution should fall with distance away from the resonance). However, the observations presented here suggest that this is not the case, but instead that the Alfvénic signature at high latitudes dominates over an extensive latitudinal region. The HF Doppler sounder is sensitive to both $\boldsymbol{E}$ and $\boldsymbol{B}$ in the ionosphere (e.g. Poole et al., 1988) which makes it a very good diagnostic for observing the modes of ULF waves. The models of Sutcliffe and Poole (1989) and Sutcliffe (1994) demonstrate that although the velocity observed by a HF Doppler sounder varies with altitude, the greatest change occurring over a very narrow altitude range corresponding to the region of peak F-region electron density. However, at these altitudes, the relative phases of the contributions from the various mechanisms are almost height independent although the absolute values are sensitive to pulsation frequency and scale size (see also Wright et al., 1997). The EISCAT and Doppler data displayed in Fig. 7 were measured at F-region heights separated by about $70 \mathrm{~km}$. EISCAT provides a measure of the true tristatic $\boldsymbol{E} \times \boldsymbol{B}$ velocity, rather than the response to a combination of mechanisms as in a Doppler sounder. The velocity observed by EISCAT would be effectively the same at the two heights as a consequence of the electric field mapping down the field lines. Thus the EISCAT and Doppler sounder velocities can be directly compared. In addition, the observed phases of the ionospheric HF Doppler, EISCAT velocity and ground magnetic signatures are consistent with predictions of how the phase of the ULF wave should vary along the field line (Hughes and Southwood, 1976).

\section{Summary}

By employing the DOPE HF Doppler sounder in conjunction with the EISCAT UHF radar, the IMAGE network of ground magnetometers and the Tromsø dynasonde, it has been possible fully to characterise the signature of a magnetospheric ULF wave both in the ionosphere and at the ground. The event, which occurred on 13 February 1996, was the result of a low- $m$ field line resonance with an unusually large scale size. Comparison of the observations with a numerical model and, for the first time, direct observation has demonstrated that the ionospheric signature of the wave was probably created by the vertical bulk motion of the ionosphere driven by the $\boldsymbol{E} \times \boldsymbol{B}$ drift associated with the pulsation electric and magnetic field perturbations. This advection of the ionospheric layers demonstrates, in this high-latitude case study, a ULF wave of Alfvénic 
character, observed some $8^{\circ}$ equatorward of the resonant field line.

This study has further highlighted the usefulness of the DOPE sounder as a high spatial and temporal resolution technique for recording the ionospheric effects of ULF waves. In addition, it is clear that experiments run in conjunction with the EISCAT UHF radar, as well as available ground magnetometers, offer the possibility of characterising various types of ULF waves and the ionospheric boundary conditions which affect them. The experimental programme SP-UKDOPE will be ideal for this purpose.

Acknowledgements. The authors wish to thank the Royal Society for funding the initial construction of the DOPE project and the Particle Physics and Astronomy Research Council for support during the work presented in this paper; Peter Chapman, Stuart Crooks and Michael Parsons, technical staff of the Radio and Space Plasma Physics group who were involved in the design and construction of the sounder; Michael Rietveld, Stein Eliassen and Thorstein Blomstrand at the EISCAT site in Ramfjordmoen for looking after the DOPE sounder and for returning data tapes to the UK; the Finnish Meteorlogical Institute and Børre Holmeslet at the Tromsø Auroral Observatory for providing magnetometer data.

Topical Editor D. Alcaydé thanks S. Buchert and F. Menk for their help in evaluating this paper.

\section{Appendix: the spatial resolution of a HF Doppler sounder}

A simplified relation for the radius of the first Fresnel zone, assuming an idealised mirror like reflection is given by (Georges, 1967)

$R=\sqrt{r_{0} \lambda}$,

where $r_{0}$ is for a vertical incidence sounder, the height of reflection and $\lambda$ is the free space wavelength of the diagnostic signal. For an F-region reflection height of $r_{0} \approx 250 \mathrm{~km}$ and a sounder frequency of $4.45 \mathrm{MHz}$ $(\lambda=67 \mathrm{~m})$ we have $R \approx 4 \mathrm{~km}$.

\section{References}

Allan, W., E. M. Poulter, and E. Nielsen, STARE observations of a Pc5 pulsation with large azimuthal wave number, J. Geophys. Res., 87, 6163, 1982.

Allan, W., E. M. Poulter, and E. Nielsen, Pc5 pulsations associated with ring current proton drifts: STARE radar observations, Planet. Space Sci., 31, 1279, 1983.

Chapman, P. J., A cost-effective frequency synthesizer for Doppler applications, Radio and Space Plasma Physics Group tech. rep. no. 60, Leicester University, 1995.

Chapman, P. J., HF Doppler receiver, Radio and Space Plasma Physics Group tech. rep. no. 63, Leicester University, 1997a.

Chapman, P. J., HF Doppler transmitter, Radio and Space Plasma Physics Group tech. rep. no. 64, Leicester University, 1997b.

Crowley, G., N. Wade, J. A. Waldock, T. R. Robinson, and T. B. Jones, High time-resolution observations of periodic frictional heating associated with a Pc5 micropulsation, Nature, 316, 528, 1985.

Crowley, G., W. J. Hughes, and T. B. Jones, Observational evidence of cavity modes in the Earth's magnetosphere, J. Geophys. Res., 92, 12233, 1987.
Davies, J. A., M. Lester, and T. R. Robinson, Deriving the normalised ion-neutral collision frequency from EISCAT observations, Ann. Geophysicae, 15, 1557, 1997.

Fenrich, F. R., J. C. Samson, G. Sofko and R. A. Greenwald, ULF high- and low- $m$ field line resonances observed with the Super Dual Auroral Radar Network, J. Geophys. Res., 100, 21535, 1995.

Fenwick, R. C., and O. G. Villard, Continuous recordings of frequency variation of the WWV-20 signal after propagation over a 4000-km path, J. Geophys. Res., 67, 3249, 1960.

Georges, T. M., Ionospheric effects of atmospheric waves, ESSA tech. rep., Inst. Environ. Res. (Boulder, Colo.), IER 57 - ITSA. 54,1967

Glangeaud, F., C. Lathuillere, M. Lambert, and Z. Y. Zhao, Pc3-4 ULF magnetic variations measured in the ionosphere by EISCAT, J. Geophys. Res., 90, 8319, 1985.

Hughes, W. J., Hydromagnetic waves in the magnetosphere, In Solar terrestrial physics, Eds. R. L. Carovillano and J. M. Forbes, D. Reidel, Dordrecht, p. 453, 1983.

Hughes, W. J., and D. J. Southwood, The screening of micropulsation signals by the atmosphere and ionosphere, J. Geophys. Res., 81, 3234, 1976.

Jacobs, J. A., and T. Watanabe, Doppler frequency changes in radio waves propagating through a moving ionosphere, Radio Sci., 1, 257, 1966.

Jacobs, J. A., Y. Kato, S. Matsushita, and V. A. Troitskaya, Classification of geomagnetic micropulsations, J. Geophys. Res., 69, 180,1964

Jarvis, M. J., and H. Gough, Digital ionosonde observations of Pc3-4 pulsations across the plasmapause, Planet. Space Sci., 36, 733, 1988.

Jarvis, M. J., and K. Morrison, Dynasonde observations of Pc1 propagation in the ionosphere, J. Atmos. Solar-Terr. Phys., 59, 817, 1997.

Kivelson, M. G., and D. J. Southwood, Hydromagnetic waves and the ionosphere, Geophys. Res. Lett., 15, 1271, 1988.

Klostermeyer, J., and J. Röttger, Simultaneous geomagnetic and ionospheric oscillations caused by hydromagnetic waves, Planet. Space Sci., 24, 1065, 1976.

Lühr, H., The IMAGE magnetometer network, STEP Int. Newslett., 4, 4, 1994.

Menk, F. W., Characterization of ionospheric Doppler oscillations in the Pc3-4 and Pi2 pulsation frequency range, Planet. Space Sci., 40, 495, 1992.

Menk, F. W., R. A. Marshall, P. W. McNabb, and I. S. Dunlop, An experiment to study the effects of geomagnetic fluctuations on ionospheric HF radio paths, J. Elec. Electr. Eng. Aust., 15, 325, 1995.

Orr, D., Magnetospheric hydrodynamic waves: their eigenperiods, amplitudes and phase variations: a tutorial introduction, J. Geophys., 55, 76, 1984.

Poole, A. W. V., P. R. Sutcliffe, and A. D. M. Walker, The relationship between ULF geomagnetic pulsations and ionospheric Doppler oscillations: derivation of a model, J. Geophys. Res., 93, 14656, 1988.

Poulter, E. M., W. Allan, J. G. Keys, and E. Nielsen, Plasmatrough ion mass densities determined from ULF pulsation eigen periods, Planet. Space Sci., 32, 1069, 1984.

Rishbeth, H., and O. K. Garriot, Introduction to ionospheric physics, Int. Geophys. Ser. 14, Academic Press, New York, London 1964.

Rishbeth, H., and P. J. S. Williams, The EISCAT ionospheric radar: the system and its early results, $Q$. J. R. Astron. Soc., 26, 478, 1985.

Ruohoniemi, J. M., R. A. Greenwald, K. B. Baker, and J. C. Samson, HF radar observations of Pc5 field line resonances in the midnight/early morning MLT sector, J. Geophys. Res., 96, 15697, 1991.

Southwood, D. J., Some features of field line resonances in the magnetosphere, Planet. Space Sci., 22, 483, 1974. 
Sutcliffe, P. R., Modelling the ionospheric signatures of geomagnetic pulsations, J. Geomagn. Geoelectr., 46, 1011, 1994.

Sutcliffe, P. R., and A. W. V. Poole, Low-latitude Pc3 pulsations and associated ionospheric oscillations measured by a digital chirp ionosonde, Geophys. Res. Lett., 11, 1172, 1984.

Sutcliffe, P. R., and A. W. V. Poole, Ionospheric Doppler and electron velocities in the presence of ULF waves, J. Geophys. Res., 94, 13505, 1989.

Sutcliffe, P. R., and A. W. V. Poole, The relationship between ULF geomagnetic pulsations and ionospheric Doppler oscillations: model predictions, Planet. Space Sci., 38, 1581, 1990.

Tedd, B. L., K. D. Cole, and P. L. Dyson, The association between ionospheric and geomagnetic pulsations in the Pc3-4 range at mid-latitudes, Planet. Space Sci., 37, 1079, 1989.

Walker, A. D. M., R. A. Greenwald, W. F. Stuart, and C. A. Green, STARE auroral radar observations of Pc5 geomagnetic pulsations, J. Geophys. Res., 84, 3373, 1979.
Wright, D. M., HF Doppler observations of ULF waves: system development and high-latitude results, $\mathrm{Ph}$. $\mathrm{D}$. thesis, University of Leicester, 1996.

Wright, D. M., T. K. Yeoman, and P. J. Chapman, High-latitude HF Doppler observations of ULF waves: 1 . waves with large spatial scale sizes, Ann. Geophysicae, 15, 1548, 1997.

Yeoman, T. K., M. Lester, D. Orr, and H. Lühr, Ionospheric boundary conditions of hydromagnetic waves: the dependence on azimuthal wave number and a case-study, Planet. Space Sci., 38, 1315, 1990.

Yeoman, T. K., D. M. Wright, T. R. Robinson, J. A. Davies, and M. T. Rietveld, High spatial and temporal resolution observations of an impulse-driven field line resonance in radar backscatter artificially generated with the Tromsø heater, Ann. Geophysicae, 15, 634, 1997. 